\title{
КОМПЛЕКСНАЯ ОЦЕНКА ЭФФЕКТИВНОСТИ СИСТЕМЫ УПРАВЛЕНИЯ ЗАТРАТАМИ НА ПРОМЫШЛЕННОМ ПРЕДПРИЯТИИ
}

\author{
(c) 2021 Бурова Екатерина Валерьевна \\ ассистент, Высшая инженерно-экономической школа, \\ Институт промышленного менеджмента, экономики и торговли \\ Санкт-Петербургский политехнический университет Петра Великого, Россия, Санкт-Петербург \\ E-mail: burova_ev@spbstu.ru
}

Статья посвящена проблеме оценки эффективности системы управления затратами на промышленном предприятии. Предложенный автором подход к решению данной проблемы учитывает сущность и особенности управления затратами на промышленном предприятии и позволяет проводить комплексную оценку эффективности системы управления затратами на всех этапах жизненного ее цикла. В исследовании в качестве критерия экономической эффективности системы выбрана модель доходности инвестиций на основе денежного потока. Для оценки влияния системы управления затратами на отдельные показатели деятельности промышленного предприятия предложены частные показатели эффективности: уровень функциональности системы, показатели качества информации и уровень развития персонала. Для реализации предложенного подхода рекомендовано использовать концепцию сбалансированной системы показателей, что обеспечит непрерывный контроль эффективности системы управления затратами.

Ключевые слова: эффективность, система управления затратами, промышленное предприятие, информационная система

\section{Введение}

По мнению исследователей, на сегодняшний день доля затрат в структуре стоимости продукции промышленных предприятий находится на уровне 80-85\% [3], что делает затраты значимым фактором конкурентоспособности предприятия [14]. Этим объясняется актуальность вопросов, связанных с совершенствованием систем управления затратами и подходами к оценке их эффективности.

В научной экономической литературе много внимания уделяется проблеме анализа и оценки эффективности систем управления [12, 13]. Все существующие подходы, по мнению Чернышева В.Ю. [12], можно разделить на 2 группы: (1) подходы, оценивающие показатели деятельности предприятия; (2) подходы, оценивающие эффективность функционирования самих систем управления. В некоторых исследованиях авторы [2, 12-14] рекомендуют комбинировать подходы первой и второй группы, что позволит оценить не только экономическую эффективность СУЗ, но и определить ее влияние на различные аспекты деятельности предприятия.

Анализ литературы показал, что на сегодняшний день общепринятой единой методики оценки эффективности систем управления за- тратами на промышленном предприятии нет. Предложенные подходы не учитывают особенности управлении затратами на промышленном предприятии и не позволяют контролировать эффективность СУЗ на всех этапах жизненного цикла.

\section{Результаты}

Для формирования подхода $\mathrm{k}$ комплексной оценке СУЗ необходимо определить ее суть и особенности управления затратами, характерные для промышленного предприятия. Проведенный анализ литературы [2, 3, 12-15], посвященный данной проблеме, позволил сформировать следующее определение СУЗ, отражающее ее сущность.

Системой управления затратами на промышленном предприятии является целевая многоуровневая информационная система сбора, подготовки, оценки, интерпретации, анализа и передачи данных о затратах предприятия, необходимых для эффективного выполнения всех функций менеджмента по всем бизнеспроекциям предприятия и по всем временным периодам управления.

На основе проведённых исследований в области управления затратами на промышленных предприятиях предлагается выделить следую- 
щие функции СУЗ: прогнозирование, планирование, учет и контроль, анализ отклонений и принятие управленческих решений. Бизнеспроекциями, характерными для промышленного предприятия, традиционно являются следующие: маркетинг, персонал, финансы, внутренние бизнес-процессы (снабжение, производство и сбыт) $[9,11]$.

Описание подхода к комплексной оценке эффективности СУЗ промышленного предприятия.

В данной статье предложен подход к комплексной оценке эффективности системы управления затратами промышленного предприятия, преимуществом которого являются: (1) сочетание оценки эффективности СУЗ, влияющей на результаты деятельности предприятия, и оценки эффективности СУЗ как информационной системы с учетом выполняемых функций, характерных для промышленного предприятия в части управления затратами [6]; (2) возможность проведения оценки эффективности системы на любой стадии жизненного цикла: от разработки до реализации.

Предложенный подход к комплексной оценке эффективности СУЗ включает в себя последовательное выполнение трех этапов:

1. Оценка экономической эффективности СУЗ, которая позволит определить влияние системы на эффективность предприятия.

Целью деятельности любого промышленного предприятия является увеличение стоимости компании, а значит, система управления затратами, внедряемая на предприятии, должна способствовать росту стоимости.

Для оценки эффективности деятельности предприятия в работе предлагается использовать модель доходности инвестиций на основе денежного потока (CFROI), которая позволит оценить изменение стоимости компании, связанное с работой СУЗ на любой стадии жизненного цикла проекта [8] Модель CFROI имеет ряд преимуществ по сравнению с другими показателями стоимости предприятия, а именно: 1) связывает измерение эффективности со способностью предприятия генерировать денежные средства [5]; 2) учитывает фактор времени при расчете денежных потоков.

Показатель CFROI рассматривает предприятие как портфель инвестиционных проектов и представляет собой внутреннюю норму доходности по уже осуществленным инвестициям [5].
Критерий создания дополнительной стоимости следующий: если показатель CFROI превышает требуемый инвесторами средний уровень, то компания создает стоимость и наоборот, если CFROI ниже требуемой доходности, то стоимость компании будет снижаться.

Кроме преимуществ модель CFROI имеет и недостатки. Как выделяют авторы [4] результат, рассчитанный по данному показателю, в итоговом виде представляет собой относительный показатель, а не сумму созданной стоимости. Вторым недостатком показателя CFROI является сложность при идентификации всех денежных потоков, как существующих, так и будущих. Для целей оценки экономической эффективности СУЗ данные ограничения не являются значимыми, поскольку в данном случае необходимо лишь убедиться, что использование СУЗ не приведет к снижению стоимости предприятия, а значит, нет необходимости в расчете абсолютного показателя стоимости. Кроме того, большинство современных промышленных предприятий используют Международные стандарты финансовой отчетности (МСФО) для оформления результатов деятельности, что облегчает расчет данных для определения всех финансовых потоков.

Целесообразность выбора показателя для оценки экономической эффективности СУЗ определяется возможностью проведения оценки не только на этапе разработки и внедрения, но и на этапе эксплуатации СУЗ, поскольку CFROI позволяет в любой момент времени рассчитать доходность инвестиций по денежному потоку, генерируемому существующими и будущими активами [5].

2. Оценка частных показателей эффективности СУЗ.

СУЗ оказывает влияние не только на финансовые результаты, но и на другие показатели деятельности промышленного предприятия. Для обеспечения полной информации об оценке эффективности СУЗ предлагается провести расчет частных показателей эффективности по следующим направлениям:

1) Оценка уровня функциональности СУЗ.

Данный показатель характеризует качество выполнения всех функций системы в процессе управления затратами. Оценку уровня функциональности системы рекомендуется провести с использованием функциональной модели оценки менеджмента (Management Functional Assessment Model - MFAM), разработанной 
Д.Масловым, П.Ватсоном и Н.Чилиши [4]. В исследовании предлагается модифицировать модель в части функций СУЗ, характерных для промышленного предприятия и оценить качество выполнения каждой выделенной функции: прогнозирование затрат, планирование затрат, учет и контроль, анализ отклонений и принятие управленческих решений [1].

2) Оценка информационной составляющей СУЗ.

СУЗ промышленного предприятия является информационной системой, а значит, качественные характеристики информации во многом определяют эффективность системы [7]. Оценка информационной эффективности может быть проведена с помощью метода групповой экспертизы на основе расчета двух показателей: достоверность информации и качество информации.

3) Оценка социальной составляющей (оценка уровня развития персонала).

Критерием социальной эффективности системы управления затратами является достижение заданного уровня по показателям, которые характеризуют отношения предприятия с персоналом, задействованным в процессе управления затратами [13]. Для проведения оценки уровня развития персонала в работе предложены такие показатели как: индекс ценности персонала, индекс вовлеченности персонала и индекс квалификации персонала.

3. Расчет интегрального показателя эффективности СУЗ на основе частных показателей.

Расчет интегрального показателя эффективности СУЗ проводится с учетом значимости частных показателей для каждого конкретного предприятия.

Алгоритм проведения комплексной оценки эффективности СУЗ на промышленном предприятии представлен на рис. 1.

Описание алгоритма расчета комплексной оценки эффективности СУЗ:

Расчет комплексной оценки эффективности проводится в три этапа:

На первом этапе производится стоимостная оценка СУЗ. Для этого по формуле (1) определяется существующий уровень показателя доходности инвестиций на предприятии на основе денежного потока $\left(\mathrm{CFROI}_{0}\right)$.

$$
\mathrm{CFROI}_{0}=\frac{\sum_{\mathrm{t}=0}^{\mathrm{T}}\left(\frac{\left(\mathrm{GCF}_{\mathrm{t}}-\mathrm{ED}_{\mathrm{t}}\right)}{\left(1+\mathrm{WACC}^{\mathrm{t}}\right.}\right)}{\sum_{\mathrm{t}=0}^{\mathrm{T}} \frac{\mathrm{GI}_{\mathrm{t}}}{(1+\mathrm{WACC})^{\mathrm{t}}}}
$$

Где $\mathrm{GCF}_{\mathrm{t}}$ - валовый денежный поток предприятия в период времени $\mathrm{t} ; \mathrm{ED}_{\mathrm{t}}-$ экономическая амортизация за период $\mathrm{t} ; \mathrm{GI}_{\mathrm{t}}-$ валовые инвестиции.

$$
\mathrm{ED}_{\mathrm{t}}=\frac{\mathrm{WACC}}{(1+\mathrm{WACC})^{\mathrm{t}}-1} *\left(\mathrm{TA}_{\mathrm{t}}-\mathrm{NDA}_{\mathrm{t}}\right)
$$

Где $\mathrm{TA}_{\mathrm{t}}-$ средняя стоимость активов компании, используемых на предприятии за период $\mathrm{t}$; $\mathrm{NDA}_{t}$ - средняя стоимость активов, не подверженная износу за период $\mathrm{t}$.

Далее рассчитывается уровень рентабельности инвестиций по денежному потоку, генерируемому СУЗ (CFROI $\left._{\text {Суз }}\right)$ :

$$
\mathrm{CFROI}_{\mathrm{Cy} 3}=\frac{\mathrm{IC}+\sum_{\mathrm{t}=1}^{\mathrm{T}} \frac{\left[\mathrm{CFO}_{\mathrm{t}}-\mathrm{CI} * \mathrm{WACC}\right]}{(1+\mathrm{WACC})^{\mathrm{t}}}}{\mathrm{IC}}
$$

Где: Т - прогнозируемый срок реализации проекта, t-год работы системы; $\mathrm{CFO}_{\mathrm{t}}-$ операционный денежный поток, связанный с функционированием СУЗ; IC - инвестиции, связанные с разработкой, внедрением, эксплуатацией СУЗ, WACC - средневзвешенная стоимость капитала.

Для признания СУЗ экономически эффективной (безубыточной), необходимо, чтобы выполнялось неравенство (4):

$$
\mathrm{CFROI}_{\mathrm{Cy}}-\mathrm{CFROI}_{\mathrm{O}} \geq 0
$$

Если неравенство не выполняется, СУЗ признается экономически неэффективной, и принимается решение об отказе от дальнейшего использования или внедрения данного варианта СУЗ.

На втором этапе рассчитываются частные показатели эффективности СУЗ:

Оценка уровня функциональности СУЗ определяется для каждой i-й функции управления затратами с помощью метода экспертных оценок. В данной работе выделено пять функций СУ3, характерных для промышленного предприятия: 1) прогнозирование затрат, 2) планирование затрат, 3) учет и контроль, 4) анализ отклонений, 5) принятие управленческих решений, и имеющих одинаковую значимость для 


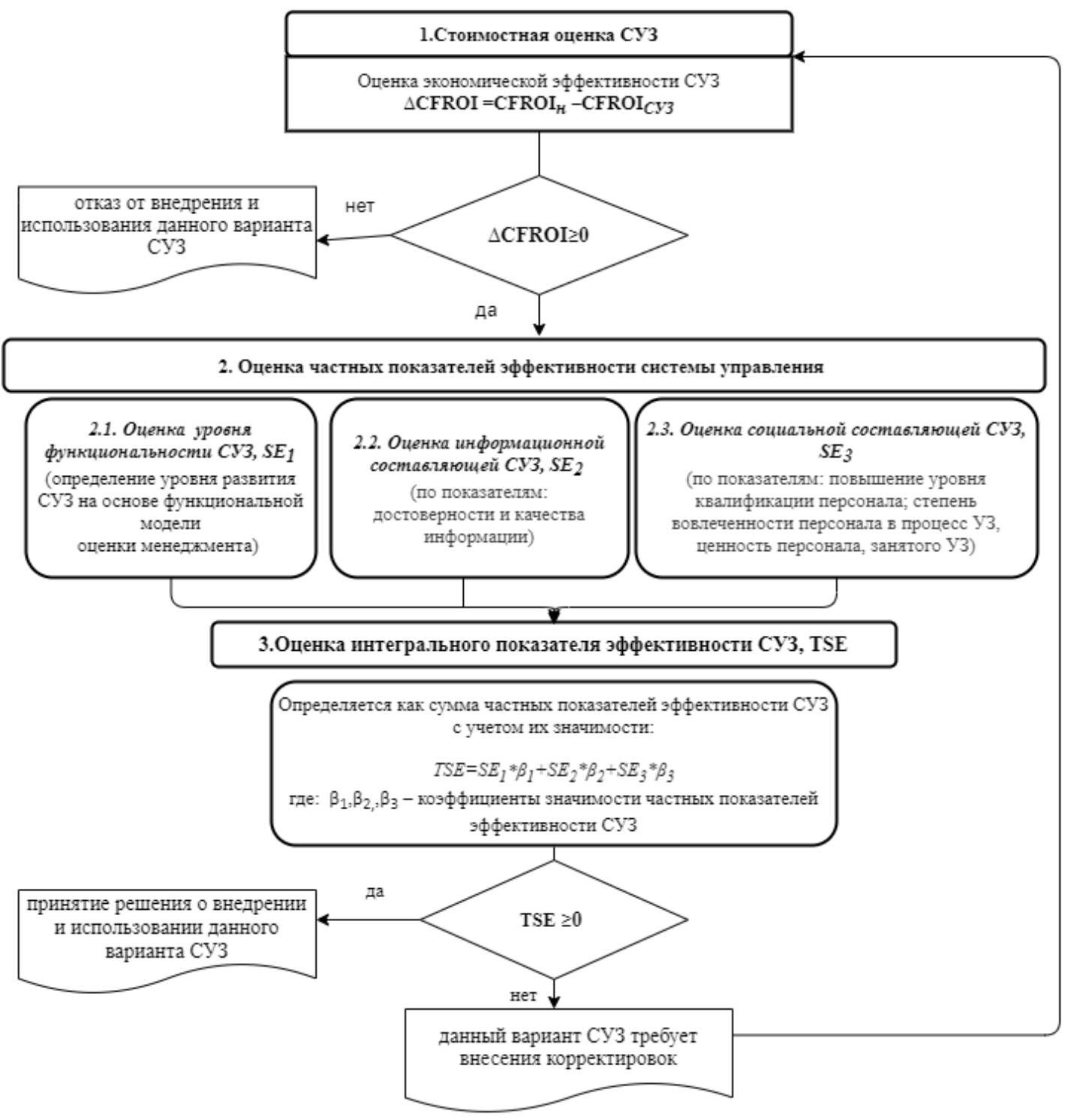

Puc. 1 Алгоритм комплексной оценки эффективности системы управления затратами промышленного предприятия.

работы системы.

Обобщенные оценки (плановые и фактические) каждой i-й функции рассчитываются аналогично как средневзвешенная величина с учетом весовых коэффициентов экспертов:

$$
f_{i}=\frac{\sum_{j=1}^{m}\left(w_{j} * f_{i j}\right)}{\sum_{j=1}^{m} w_{j}}
$$

где: $w_{j}$ - весовой коэффициент j-эксперта; $\mathrm{f}_{\mathrm{ij}}$ - оценка j-м экспертом i-й функции СУЗ.

Оценка каждой i-й функции управления $\left(\mathrm{f}_{\mathrm{ij}}\right)$ определяется экспертами с использованием универсальной вербально-числовой шкалы Харрингтона [10] и будет находиться в интервале $[0 ; 1]$.
Далее определяется общий уровень функциональной эффективности СУЗ по формулам (6) и (7):

$$
\begin{aligned}
& \mathrm{F}^{0}=\frac{1}{5} \sum_{\mathrm{i}=1}^{5} \mathrm{f}_{\mathrm{i}}^{0} \\
& \mathrm{~F}^{\mathrm{CY} 3}=\frac{1}{5} \sum_{\mathrm{i}=1}^{5} \mathrm{f}_{\mathrm{i}}^{\mathrm{CY3}}
\end{aligned}
$$

Где F0 - планируемый уровень функциональности СУЗ; FСУЗ - фактическая оценка уровня функциональности оцениваемой СУЗ, $\mathrm{f}_{\mathrm{i}}^{0}, \mathrm{f}_{\mathrm{i}}^{\mathrm{Cу} 3}$ соответственно плановые и фактические оценки і-й функции СУЗ. 
На основе полученных оценок уровня функциональности рассчитывается первый частный показатель эффективности СУЗ $\mathrm{SE}_{1}$.

$$
\mathrm{SE}_{1}=\left\{\begin{array}{cc}
1-\frac{\mathrm{F}^{0}}{\mathrm{~F}^{\mathrm{C} 3},}, & \text { при }\left\{\begin{array}{c}
\mathrm{F}^{0} \neq 0 \\
\mathrm{~F}^{\mathrm{Cу}}>\mathrm{F}^{0}
\end{array}\right. \\
\frac{\mathrm{F}^{\mathrm{Cy}}}{\mathrm{F}^{0}}-1, & \text { при }\left\{\begin{array}{c}
\mathrm{F}^{\mathrm{C}} \mathrm{F}^{\mathrm{Cy}} \neq 0 \\
\mathrm{~F}^{\mathrm{Cy} 3}<\mathrm{F}^{0}
\end{array}\right. \\
0, & \text { при } \mathrm{F}^{\mathrm{Cу} 3}=\mathrm{F}^{0}
\end{array}\right.
$$

Показатель уровня функциональности СУЗ принимает значения в интервале $\mathrm{SE}_{1} \in[-1 ; 1]$. При $-1 \leq \mathrm{SE}_{1}<0$ СУЗ признается неэффективной по данному показателю; при $0 \leq \mathrm{SE}_{1} \leq 1$ СУЗ считается эффективной по уровню функциональности.

Оценка информационной составляющей эффективности СУЗ проводится на основе двух показателей: уровень достоверности и качество информации.

Уровень достоверности информации определяется с помощью подхода, предложенного в [10]: на основе шкалы Кента [16].

Далее рассчитывается плановое значение степени достоверности информации $\left(\mathrm{D}^{0}\right)$ и значение степени достоверности информации данного варианта СУЗ (DCУ3) по формулам (9) и (10):

$$
\begin{gathered}
\mathrm{D}^{0}=\frac{\sum_{\mathrm{j}=1}^{\mathrm{m}} \mathrm{w}_{\mathrm{j}} * \mathrm{D}_{\mathrm{j}}^{0}}{\sum_{\mathrm{j}=1}^{\mathrm{m}} \mathrm{w}_{\mathrm{j}}} \\
\mathrm{D}^{\mathrm{Cy} 3}=\frac{\sum_{\mathrm{j}=1}^{\mathrm{m}} \mathrm{w}_{\mathrm{j}} * \mathrm{D}_{\mathrm{j}}^{\mathrm{Cy3}}}{\sum_{\mathrm{j}=1}^{\mathrm{m}} \mathrm{w}_{\mathrm{j}}}
\end{gathered}
$$

где: $\mathrm{w}_{\mathrm{j}}$ - весовой коэффициент j-го эксперта; $\mathrm{D}_{j}^{0}$ - оценка j-м экспертом приемлемого уровня достоверности информации для принятия УР в оцениваемой СУЗ; $\mathrm{D}_{\mathrm{j}}^{\mathrm{CV} 3}$ - оценка $\mathrm{j}$-м экспертом уровня достоверности информации для принятия УР в оцениваемой СУЗ.

Оценку качества информации в исследовании предлагается провести по таким параметрам, как репрезентативность, достаточность, актуальность и своевременность, с использованием экспертной оценки и шкалы Кента. Окончательные оценки качества информации СУЗ $\left(\mathrm{K}^{0}\right.$ - планируемое значение, $\mathrm{K}^{\mathrm{CУ} 3}$ - для данного варианта СУЗ) определяются аналогично оценкам уровня достоверности информации с учетом весовых коэффициентов компетентности экспертов.

Оценка информационной составляющей СУЗ рассчитывается как среднеарифметическое значение по формулам (11) и (12):

$$
\begin{aligned}
& \mathrm{R}^{\mathrm{o}}=\frac{\mathrm{D}^{0}+\mathrm{K}^{0}}{2} \\
& \mathrm{R}^{\mathrm{Cy} 3}=\frac{\mathrm{D}^{\mathrm{Cy} 3}+\mathrm{K}^{\mathrm{Cy} 3}}{2}
\end{aligned}
$$

Где $\mathrm{R}^{\mathrm{o}}$ - планируемый уровень качества информационной составляющей в СУЗ, RСУ3 фактический уровень качества информационной составляющей данного варианта СУЗ.

Тогда частный показатель эффективности СУЗ по информационной составляющей $\left(\mathrm{SE}_{2}\right)$, может быть рассчитан по следующей формуле (13):

$$
\mathrm{SE}_{2}=\left\{\begin{array}{cc}
1-\frac{\mathrm{R}_{0}}{\mathrm{R}_{\mathrm{H}}}, & \text { при }\left\{\begin{array}{c}
\mathrm{R}_{0} \neq 0 \\
\mathrm{R}_{\mathrm{H}}>\mathrm{R}_{0}
\end{array}\right. \\
\frac{\mathrm{R}_{\mathrm{H}}}{\mathrm{R}_{0}}-1, & \text { при }\left\{\begin{array}{c}
\mathrm{R}_{\mathrm{H}} \neq 0 \\
\mathrm{R}_{\mathrm{H}}<\mathrm{R}_{0}
\end{array}\right. \\
0, & \text { при } \mathrm{R}_{\mathrm{H}}=\mathrm{R}_{0}
\end{array}\right.
$$

Рассчитанный показатель эффективности СУЗ принимает значения в интервале $\mathrm{SE}_{2} \in[-1 ; 1]$. При $-1 \leq \mathrm{SE}_{2}<0$ СУЗ признается неэффективной по данному показателю; при $0 \leq \mathrm{SE}_{2} \leq 1$ СУЗ считается эффективной по критерию качества информации.

Для проведения оценки социальной составляющей, связанной с развитием персонала, вовлеченного в процесс управления затратами, автором предлагается использовать следующие частные показатели, представленные в табл. 3 .

Далее определяется общая оценка уровня развития персонала (Р) (14):

$$
\mathrm{P}=\frac{\mathrm{I}_{\mathrm{c}}+\mathrm{I}_{\mathrm{k}}+\mathrm{I}_{\mathrm{q}}}{3}
$$

На основе данной оценки по формуле (15) рассчитывается частный показатель эффективности СУЗ, связанный с оценкой уровня развития персонала $\left(\mathrm{SE}_{3}\right)$ :

$$
\mathrm{SE}_{3}=\left\{\begin{array}{cc}
1-\frac{1}{\mathrm{P}}, & \text { при } \mathrm{P}>1 \\
\mathrm{P}-1, & \text { при } \mathrm{P}<1 \\
0, & \text { при } \mathrm{P}=1
\end{array}\right.
$$

Показатель оценки уровня развития пер- 
Таблица 3. Показатели для расчета эффективности социальной составляющей СУЗ.

\begin{tabular}{|c|c|c|}
\hline $\begin{array}{c}\text { Оценка } \\
\text { эффективности } \\
\text { социальной } \\
\text { составляющей СУЗ }\end{array}$ & $\begin{array}{c}\text { Название } \\
\text { показателя }\end{array}$ & Формула для расчета \\
\hline $\begin{array}{c}\text { Оценка ценности } \\
\text { персонала, занятого } \\
\text { У3 }\end{array}$ & $\begin{array}{c}\text { Индекс } \\
\text { ценности } \\
\text { персонала }\end{array}$ & $\begin{array}{c}\mathrm{I}_{\mathrm{c}}=\frac{3 \Pi^{\text {суз }}}{3 \Pi^{0}} \\
3 \Pi^{0}, 3 \Pi^{\text {суз }} \text {-плановый и фактический средний размер зарплаты } \\
\text { персонала, занятого управлением затратами }\end{array}$ \\
\hline $\begin{array}{c}\text { Оценка } \\
\text { вовлеченности } \\
\text { персонала в процесс } \\
\text { у3 }\end{array}$ & $\begin{array}{c}\text { Индекс } \\
\text { вовлеченности } \\
\text { персонала }\end{array}$ & $\begin{array}{c}\mathrm{I}_{\mathrm{k}}=\frac{\mathrm{K}_{\mathrm{y3}}^{\mathrm{cУ3}} * \mathrm{~K}_{\mathrm{cп}}^{0}}{\mathrm{~K}_{\mathrm{cп}}^{\mathrm{CУ}} * \mathrm{~K}_{\mathrm{y3}}^{0}} \\
\mathrm{~K}_{\mathrm{y} 3}^{0}, \mathrm{~K}_{\mathrm{y3}}^{\mathrm{Cу3}}-\text { плановое и фактическое количество сотрудников, } \\
\text { занятых управлением затратами; } \mathrm{K}_{\mathrm{cп}}^{0}, \mathrm{~K}_{\mathrm{cп}}^{\mathrm{Cу3}}-\text { плановая и } \\
\text { фактическая среднесписочная численность сотрудников } \\
\text { предприятия }\end{array}$ \\
\hline $\begin{array}{c}\text { Оценка уровня } \\
\text { квалификации } \\
\text { персонала, занятого } \\
\text { у3 }\end{array}$ & $\begin{array}{c}\text { Индекс } \\
\text { квалификации } \\
\text { персонала }\end{array}$ & 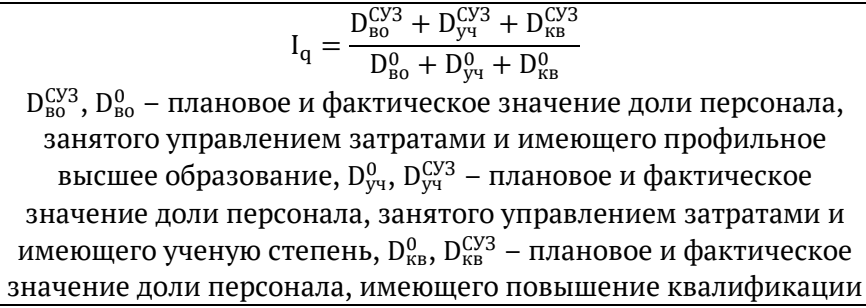 \\
\hline
\end{tabular}

сонала принимает значения в интервале $\mathrm{SE}_{3} \in[-1 ; 1]$. . уровень развития персонала неудовлетворительный; при $0 \leq \mathrm{SE}_{3} \leq 1-\mathrm{CУ}$ по данному показателю признается эффективной.

На третьем этапе комплексной оценки эффективности определяется интегральный показатель эффективности СУЗ на основе частных показателей, рассчитанных на втором этапе (16):

$$
\mathrm{TSE}=\mathrm{SE}_{1} * \beta_{1}+\mathrm{SE}_{2} * \beta_{2}+\mathrm{SE}_{3} * \beta_{3}
$$

Где $\beta_{1}, \beta_{2}, \beta_{3}-$ коэффициенты значимости частных показателей эффективности функционирования СУЗ, определенные с помощью экспертного метода ранжирования. Интегральный показатель эффективности СУЗ может принимать значения в интервале TSE $\in[-1 ; 1]$.

По результатам расчета интегрального показателя эффективности при условии безубыточности данного варианта СУЗ принимается окончательное решение:

1) При TSE $\geqslant 0$ данный вариант СУЗ признается приемлемым и принимается решение о внедрении и использовании;

2) При TSE < 0 данный вариант СУЗ считается недостаточно эффективным, для принятия решения о его внедрении и использовании требуется внесение корректировок с целью улучшения частных показателей эффективности.

Для реализации предложенного подхода к комплексной оценке эффективности системы управления затратами на промышленном предприятии целесообразно использовать концепцию сбалансированной системы показателей (BSC), поскольку выделенные направления оценки эффективности системы полностью соответствуют перспективам концепции BSC (рис. 2).

Использование концепции сбалансированной системы показателей обеспечит контроль эффективности СУЗ на стадии ее разработки, внедрения и эксплуатации.

\section{Заключение.}

Предложенный в статье методический подход к оценке эффективности системы управления затратами промышленного предприятия позволит: (1) проводить анализ СУЗ в различных аспектах: финансовом, социальном, информационном и функциональном, что обеспечит комплексность оценки и соответствие основным бизнес-проекциям промышленного предприятия и перспективам BSC; (2) выполнять оценку эффективности на всех этапах жизненного цикла системы управления затратами: от стадии разработки до окончания реализации проекта. Кроме того, частные показатели, выбранные для проведения оценки, могут меняться исходя из целей развития и специализации промышленного предприятия, что подтверждает универсальность подхода.

В ходе дальнейших исследований предло- 


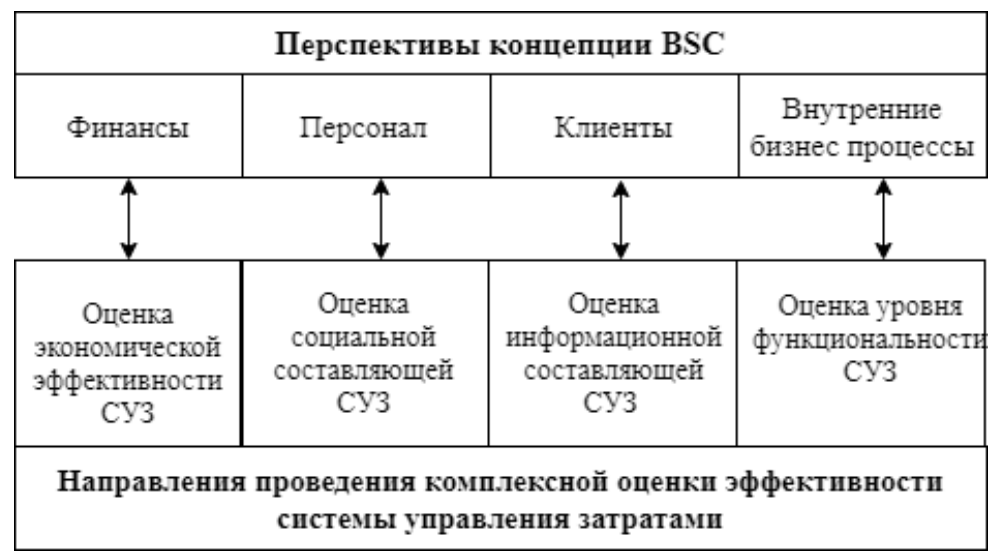

Puc. 2. Комплексная оценка эффективности СУз в концепции BSC.

женная методика комплексной оценке эффективности СУЗ потребует доработки и уточнений в части ее организации, выбора конкретных показателей и инструментов, но сам предложенный подход позволит своевременно выявлять слабые места в системе управления затратами и принимать решения по ее совершенствованию, что позволит повысить эффективность деятельности предприятия в целом.

\section{Библиографический список}

1. Гришунин С. В., Муханова Н. В., Сулоева С. Б. Разработка концепции риск-контроллинга для промышленного предприятия // Организатор производства. 2018. № 1 (26).

2. Коломеец Е. А. Оценка эффективности системы управления затратами // Наука и производство Урала. 2015. № 11. С. $165-169$.

3. Лабзунов П.П. Методология управления затратами промышленного предприятия в современном обществе // Российский технологический журнал. 2017. № 2 (5). С. 70-77.

4. Маслов Д. В., Ватсон П., Чилиши Н. Диагностическая самооценка системы управления по критериям функциональная модели // Качество. Инновации. Образование. 2005. № 3. С. 18-22.

5. Назарова В. В., Бирюкова Д. С. Стоимостные методы оценки эффективности менеджмента компании // Научный журнал НИУ ИТМО. Серия «Экономика и экологический менеджмент». 2014. № 3.

6. Родионов Д. Г., Конников Е.А., Алферьев Д.А. Информационный капитал предприятия как целевой показатель развития в рамках цифровых экономических систем // Экономические науки. 2020. № 190. С. 131-137.

7. Родионов Д. Г., Рудская И. А. Стратегическое управление финансами предприятия // Российский экономический интернет-журнал. 2018. № 4. С. 95.

8. Родионов Д. Г., Ялымов С. В., Сергеев Д. А. Фискальный инструмент управления развитием сектора малого и среднего предприниматель ства // Экономические науки. 2020. № 189. С. 92-98.

9. Рудская И.А., Родионов Д.Г. Методические принципы и этапы формирования финансовой стратегии предприятия // Kant. 2018. № 4 (29).

10. Самохвалов Ю.Я. Оценка обоснованности управленческих решений на основе нечеткой логики // Управляющие системы и машины. 2017.

11. Сулоева С. Б. Стратегический контроллинг на промышленном предприятии: Теория, методология, инструментарий // СПб.: Нестор. 2005.

12. Чернышев В. Ю. Методические подходы к оценке эффективности управления предприятием // Экономинфо. 2010. № 13.

13. Шавкун Г.А.Подход к оценке эффективности функционирования системы управления затратами промышленного предприятия // Вестник Воронежского государственного университета. Серия: Экономика и управление. 2017. № 1. С. 29-34.

14. Davila A., Foster G., Jia N. The valuation of management control systems in start-up companies: international field-based evidence // European Accounting Review. 2015. № 2 (24). C. 207-239.

15. Ditkaew K. The Effects of Cost Management Quality on the Effectiveness of Internal Control and Reliable DecisionMaking: Evidence from Thai Industrial Firms Scientia Moralitas Research Institute, 2018.C. 60-69.

16. Kent S. Strategic intelligence for American world policy / S. Kent, Princeton University Press, 2015. 